\title{
Modèles probabilistes en hydrologie
}

\author{
par J.-R. Tiercelin \\ Ingénieur du Génie Rural, des Eaux et des Forêts \\ Division Hydrologie du C.T.G.R.E.F."
}

Un article paru dans la présente revue, sous la signature de MM. Mougin et Dujardin [6], a montré l'intérêt qui s'attache à la conception et à l'utilisation des modèles en hydrologie, et plus précisément des modèles dits déterministes, conceptuels ou physiques. En complément de l'information constituée par cet article, les lignes qui suivent ont pour objet d'attirer l'attention sur un autre type de modèles hydrologiques également susceptibles d'applications pratiques: les modèles probabilistes ou stochastiques.

\section{Consistance des modèles probabilistes}

Un problème relatif à l'aménagement des eaux, présenté de façon schématique, va permettre de mettre en évidence la raison d'être et la consistance d'un modèle probabiliste très simple.

Supposons qu'il s'agisse de concevoir la régularisation d'un cours d'eau au moyen d'une retenue, en vue de garantir à l'aval un débit fixé à l'avance avec une certaine probabilité de défaillance.

$\mathrm{Si}$, par chance, le projeteur dispose de plusieurs dizaines d'années d'observations de débits, il lui suffira généralement de simuler le fonotionnement de la réserve à partir des seuls débits observés, pour apprécier la fréquence des défaillances de la fourniture d'eau par rapport à la de-

* Centre Technique du Génie Rural, des Eaux et des Forêts (ex. C.E.R.A.F.E.R.), Parc de Tourvoie, 92160 Antony. mande, et choisir ainsi le volume de retenue susceptible d'apporter la garantie demandée.

Malheureusement, des circonstances aussi favorables sont rares, et en appliquant une telle méthode avec des séries hydrologiques trop brèves, le problème peut être insoluble ou donner lieu à une solution d'une imprécision inacceptable (comment, par exemple, apprécier de cette façon une défaillance de récurrence décennale à partir d'une série inférieure à dix ans?). Une solution au problème consistera à établir tout d'abord une représentation schématique des données hydrologiques utilisées. Supposons, comme cela se fait souvent pour ce type de problème, qu'il soit jugé d'une précision suffisante de remplacer la série des débits par la séquence des débits moyens mensuels correspondants. Il apparaît alors que, si les débits mensuels sont représentés par :

- douze lois de probabilités (établies à partir des distributions de fréquence des débits des douze mois de l'année);

- douze paramètres ou jeux de paramètres (définissant la liaison statistique entre les débits de chaque mois de l'année et les débits des mois antérieurs),

l'ensemble des formulations mathématiques précédentes, qui rend compte à la fois de la fréquence d'apparition des différents débits étudiés et de leur enchaînement dans le temps, donne une représentation des débits observés qui est suffisamment complète au regard du problème posé.

Un tel ensemble de formulations mathématiques, essentiellement probabilistes, destiné à schématiser un phénomène réel, constitue par définition un modèle probabiliste.

Le modèle étant établi, il s'agit dans une deuxième phase de l'exploiter pour résoudre le problème posé. Deux possi- 
bilités se présentent. Dans certains cas particuliers relativement simples, l'application de calculs statistiques permettra parfois d'obtenir la réponse cherchée [2]. Néanmoins, le plus souvent, on ne connaîtra aucune possibilité de résolution analytique, et on procèdera alors par tirage au hasard, suivant les méthodes dites de Monte-Carlo [4] [2], afin de reconstituer artificiellement une longue série de débits qui auraient pu réellement s'observer à la station considérée. On ne prétend pas ainsi créer de l'information; il s'agit uniquement d'un artifice destiné en quelque sorte à dilater l'information disponible pour se retrouver, sur le plan des techniques de calcul, dans le cas précédemment évoqué où l'on disposait d'une très longue série d'observations réelles de débits, permettant une simulation $\mathrm{du}$ fonctionnement hydraulique de l'ouvrage projeté. Bien entendu, il faudra s'être assuré, avant utilisation, que le modèle est suffisamment fidèle aux débits qu'il est censé représenter.

\section{Quelques exemples concrets}

Après avoir montré sur un exemple volontairement simplifié ce que pouvait être un modèle probabiliste très élémentaire, il paraît utile de préciser, par des exemples réels d'utilisation, les possibilités des modèles de ce type.

\section{Régularisation de la Doller par le barrage de Michelbach. aval}

La Direction départementale de l'Agriculture du HautRhin et l'Agence du Bassin Rhin-Meuse étudient un projet pour la régularisation de la Doller. Le problème posé consiste à concevoir un aménagement, essentiellement constitué par le barrage de Michelbach-aval, qui permette de prélever de l'eau dans la Doller lorsque le débit dépasse $2,75 \mathrm{~m}^{3} / \mathrm{s}$, et d'en restituer lorsque le débit descend en dessous de $1 \mathrm{~m}^{3} / \mathrm{s}$. Il s'agit de déterminer, en fonction des caractéristiques de la prise d'eau alimentant le barrage, la fréquence et l'importance des défaillances du système vis-àvis de la garantie recherchée.

La situation est complexe sur le plan de la confrontation des ressources et des besoins. En effet, il est possible d'observer, en toutes saisons, des débits inférieurs à $1 \mathrm{~m}^{3} / \mathrm{s}$, nécessitant de faire appel à l'eau stockée derrière le barrage, et des débits supérieurs à $2,75 \mathrm{~m}^{3} / \mathrm{s}$, permettant de réalimenter la réserve, le passage d'une situation à l'autre pouvant s'effectuer en l'espace de quelques jours. Dans ces conditions, on ne peut recourir à aucune des deux simplifications consistant soit à travailler avec les seules moyen nes mensuelles, soit à considérer les débits de la seule période estivale. Il est nécessaire de s'intéresser aux débits journaliers de toute l'année pour une estimation correcte des risques de défaillance.

Les données hydrologiques essentielles sont constituées par une série de 9 ans de débits observés à la station de Masevaux. Cette période est beaucoup trop courte pour que l'on puisse avoir une idée 'des défaillances ayant une période de récurrence de l'ordre de 10 ou 20 ans, ce qui oblige à envisager l'utilisation d'un modèle et la simulation des débits.

Il y avait lieu, par ailleurs, de se demander dans quelle mesure l'information pluviométrique régionale pouvait être utilisée pour améliorer la connaissance des débits. Cette amélioration ne pouvait être espérée qu'avec une ou plusieurs séries pluviométriques présentant les caractéristiques suivantes:

- période d'observation commune avec celle des débits, afin de connaître la relation entre les deux grandeurs;

- période d'observation extérieure à celle des débits, aussi longue que possible, pendant laquelle on cherche à reconstituer, grâce à la relation précédente, les débits non observés.

La station de Sewen, pour laquelle 70 ans de relevés étaient disponibles, répondait à cette double exigence.

Bien entendu, la pluie 'de cette station unique n'a qu'un rapport assez lâche avec la lame d'eau moyenne tombant sur le bassin versant de $120 \mathrm{~km}^{2}$ dominant l'emplacement prévu pour la prise d'eau. La liaison entre pluies et débits ne pouvait être alors envisagée qu'en termes statistiques, mais compte tenu de la faible représentativité de la station pluviométrique, et quoique l'on ait utilisé des débits journaliers pour la simulation, la recherche d'une liaison pluies-débits à l'échelle journalière ne pouvait être envisagée; c'est une corrélation mensuelle qui a été simplement retenue.

En définitive, les opérations successives ont été les suivantes, de façon schématique:

- établissement des régressions statistiques des débits mensuels à Masevaux par rapport aux pluies mensuelles à Sewen;

- simulation, à l'aide des formules de régression et des méthodes de Monte-Carlo, de 50 années de débits mensuels à Masevaux, à partir des pluies réellement observées à Sewen pour la période 1923-1972;

- calage d'un modèle de débits journaliers sur les données de Masevaux, assorties de coefficients multiplicateurs saisonniers pour représenter les débits au droit de la prise;

- simulation, à l'aide du modèle, de 50 années de débits journaliers au droit de la prise d'eau;

- application aux débits journaliers obtenus, de coefficients permettant de respecter les débits moyens mensuels issus de la simulation pluies-débits.

La simulation hydrologique ainsi décrite est suivie d'une simulation du fonctionnement hydraulique de l'aménagement, permettant de tester différentes hypothèses relatives à la prise d'eau, en vue de déterminer celle qui apporte de façon la plus économique le niveau de garantie recherché.

\section{Aménagement de la moyenne vallée de l'Eyrieux :}

Le Syndicat Mixte d'Equipement du département de l'Ardèche étudie un projet d'aménagement de la vallée de l'Eyrieux, dont l'opération principale est constituée par la construction du barrage du Cheylard. La question posée est de déterminer les conditions économiques de cette création, destinée notamment à améliorer la satisfaction des besoins d'irrigation.

Pour traduire en termes économiques les conséquences de l'aménagement, il est nécessaire de simuler son fonc- 

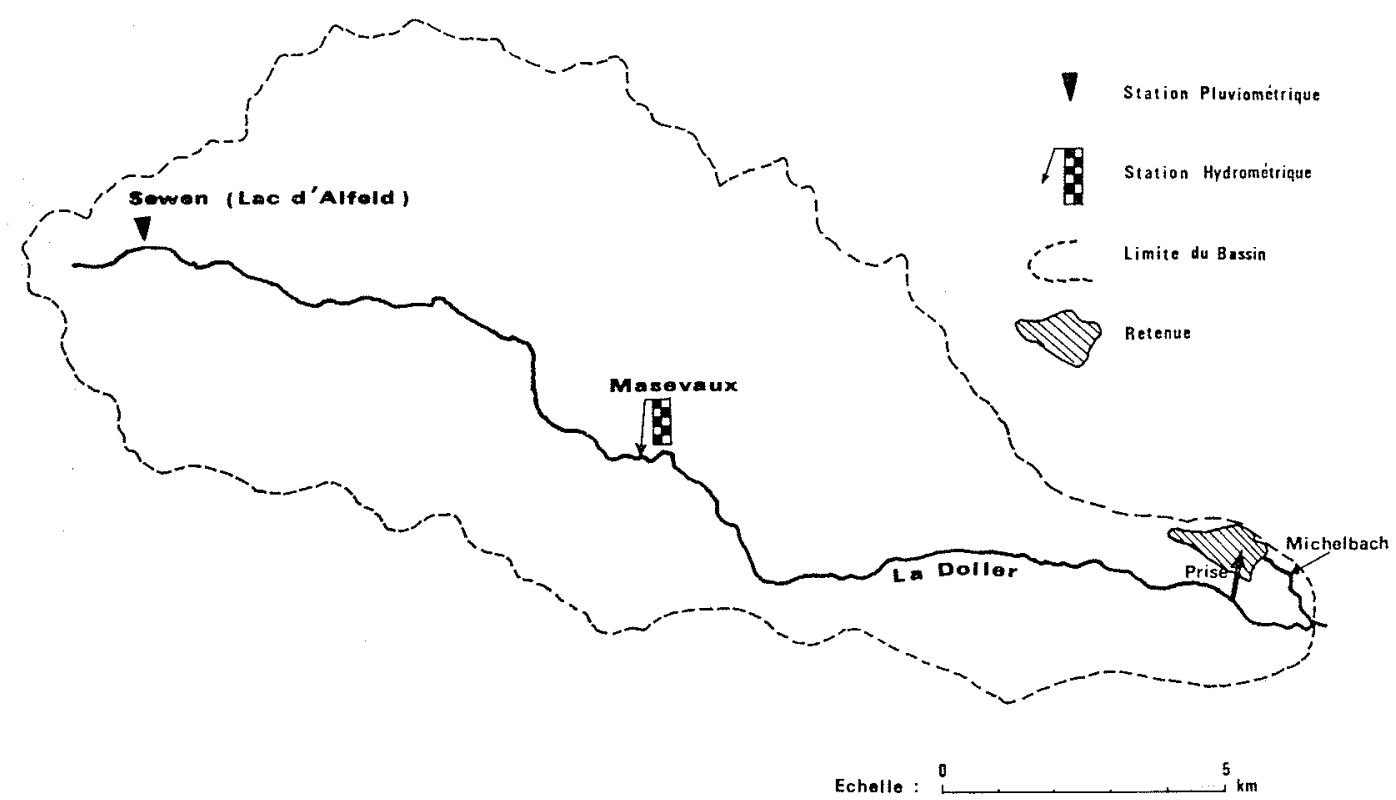

II

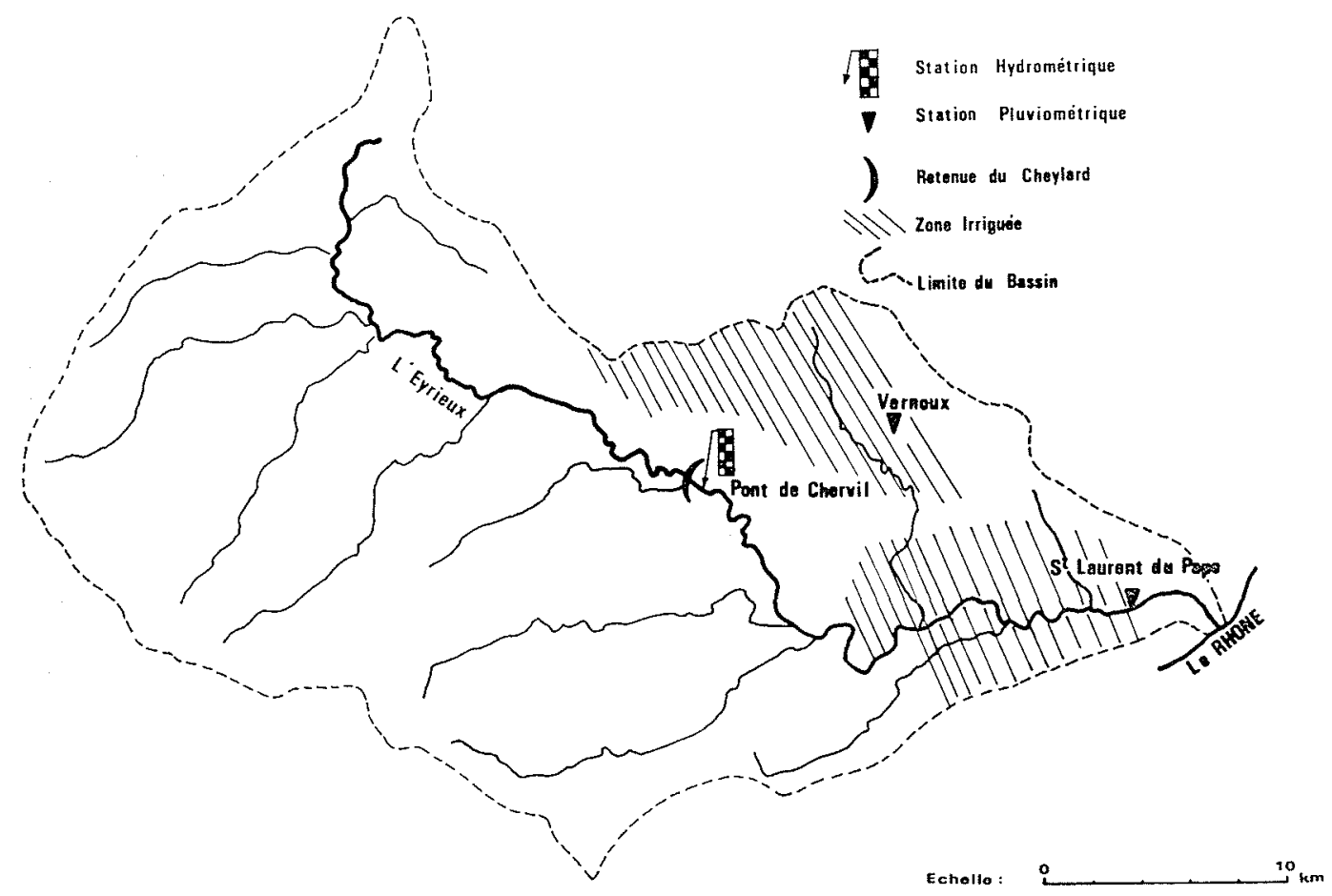

2/ 
tionnement pendant la durée choisie comme période d'amortissement (25 ans), de façon à faire ressortir la succession des défaillances du système vis-à-vis de la demande, défaillances qui seront ensuite traduites en pertes de rendement.

Grâce à quelques hypothèses simplificatrices, le maîtred'œuvre a pu effectuer cette simulation, sans recourir à un modèle, à partir de débits réellement observés à Pont-deChervil, pour la période d'amortissement choisie. Cependant, il faut voir que le résultat économique attendu pour les 25 ans qui suivront la mise en service des ouvrages est, a priori, un résultat aléatoire qui dépend de la succession des années sèches et humides à l'intérieur de la période d'amortissement: il a donc été décidé de simuler un assez grand nombre de séries de 25 ans et d'effectuer à la fin le dépouillement statistique des résultats économiques obtenus à partir de chacune de ces séries.

Si la ressource en eau est variable, les besoins à satisfaire le sont aussi puisqu'il s'agit d'irrigation. En première approximation, ressources et besoins varient en fonction de la pluviométrie. Il est indispensable de rendre compte correctement de la liaison qui en découle entre ressources et besoins, si on veut estimer correctement les défaillances tant en ce qui concerne leur probabilité d'apparition que leur importance. La simulation effectuée porte donc à la fois sur les débits alimentant le futur réservoir (station de Pont-de-Chervil), et sur les pluies alimentant les futurs périmètres d'irrigation (stations de Vernoux-en-Vivarais et de Saint-Laurent-du-Pape).

On conçoit bien que, dans ces conditions, la relation entre pluies et débits, comme dans l'exemple précédent, soit floue et ne puisse être prise en compte qu'en termes statistiques.

Les opérations effectuées successivement ont été les suivantes, de façon schématique:

- établissement des régressions statistiques des débits décadaires à Pont-de-Chervil, par rapport aux pluies décadaires à Vernoux et Saint-Laurent, en tenant compte de l'autocorrélation des débits décadaires successifs;

- simulation de séries de pluies et débits décadaires concomittants à l'aide des relations statistiques cidessus et des méthodes de Monte-Carlo;

- simulation du système hydraulique, constitué essentiellement par les deux réservoirs que sont la retenue du Cheylard et le sol des périmètres irrigués, en vue de faire apparaître la succession des défaillances;

- calcul des résultats économiques dans différentes hypothèses d'aménagement.

\section{Autres domaines d'application des modèles probabilistes}

L.es exemples ci-dessus ne doivent pas laisser croire que, seuls, les aménagements ponctuels justifient l'utilisation de modèles probabilistes: les méthodes d'analyse multivariate permettent d'aborder la conception d'aménagements complexes, comportant notamment des réserves multiples [3].

En outre, nous n'avons abordé que le domaine de la conception des aménagements. En fait, si on prend le terme de modèle dans son sens le plus large, il apparaît, par exemple, qu'une série de relations de régression multilinéaire pour la prévision des apports en eau [5] constitue également un modèle probabiliste, qui présente en particulier les avantages suivants: il donne des prévisions qui peuvent être graduées en probabilité, et permet d'exploiter des données très diverses par leur nature et par l'empla. cement des stations d'observations.

\section{Intérêt des modèles probabilistes}

Les exemples présentés induisent un certain nombre de considérations générales sur la raison d'être et l'intérêt des méthodes probabilistes, et notamment des modèles probabilistes, en hydrologie.

\section{Les problèmes à résoudre}

Tout d'abord, divers problèmes relatifs à l'aménagement des eaux doivent être posés en termes probabilistes. Ainsi, par exemple, il y a toujours une certaine probabilité, fûtelle très faible, pour que la satisfaction des demandes en eau par un aménagement des ressources soit tenue en échec. Dans les objectifs de l'aménagement, il faut donc assortir les demandes en eau d'une probabilité de nonsatisfaction.

Pour qu'on puisse, dans ce contexte, accorder quelque crédit aux résultats des calculs et simulations, il faut que l'outil hydrologique utilisé respecte non seulement, ce qui est évident et généralement assez facile à obtenir, l'ordre de grandeur des débits utilisés, mais également, et ceci est tout aussi important, la dispersion des valeurs qu'ils peuvent prendre et, dans une moindre mesure, la liaison entre des débits se succédant dans le temps. En outre, dans le cas complexe, constitué notamment par la satisfaction des besoins d'irrigation, où les besoins sont variables euxaussi, il faut respecter la liaison existant entre les ressources et les besoins qui se manifestent simultanément.

En résumé, il est essentiel de respecter les caractéristiques statistiques des séries hydrologiques (et éventuellement de la fluctuation de la demande en eau) qui nous intéressent: pour ce faire, il est tout à fait adéquat d'utiliser un modèle probabiliste, qui est bâti précisément à partir des caractéristiques statistiques en question.

\section{L'information hydrologique disponible}

Dans tout problème faisant appel à l'hydrologie, il y a lieu de considérer, parallèlement à la définition du problème lui-même, la nature des données disponibles pour le résoudre.

Dans beaucoup de cas d'application (tel que celui de la Doller), la seule information rigoureusement indispensable est constituée par quelques années d'observations de débits en une station. Ainsi, l'utilisation d'un modèle probabiliste permet de tirer éventuellement parti d'une information relativement réduite.

Le plus souvent, la situation se présente de 'façon moins défavorable et il est possible d'exploiter une information hydrologique complémentaire. Tout d'abord, de façon courante, celle-ci peut être constituée par l'information pluviométrique. Lorsqu'il existe une ou plusieurs séries pluvio- 
métriques présentant à la fois une relation suffisante avec les débits observés et une durée d'observation supérieure à celle de la série hydrométrique, les méthodes probabilistes permettent d'introduire l'information pluviométrique pour améliorer la connaissance des débits (exemple de l'aménagement de la Doller), ce qui valorise d'ailleurs les longues séries d'observations pluviométriques qui existent un peu partout sur le territoire.

Un autre type d'information complémentaire utile est constitué par les séries hydrométriques de longue durée relatives à des bassins versants présentant une certaine analogie avec le bassin qui intéresse le projeteur. En effet, d'une part le fait de pouvoir se référer à des séries d'observations suffisamment longues est fort utile pour le choix des lois de probabilité et de relations statistiques dignes de foi. D'autre part, ce type de démarche peut conduire à l'estimation de certains paramètres statistiques utilisables pour les modèles probabilistes: la référence à un réseau de stations hydrométriques anciennes permet d'obtenir notamment une précision très intéressante dans l'estimation de la variabilité relative des débits [7].

D’une façon générale, les modèles probabilistes permettent d'exploiter une information extrêmement variable, même lorsque celle-ci représente très mal les phénomènes physiques en jeu, ce qui est souvent le cas en pratique. Des considérations de cet ordre sont d'ailleurs à l'origine de l'introduction des méthodes statistiques en hydrologie [3].

En contrepartie des avantages présentés par les méthodes statistiques, il faut observer qu'elles exigent des données d'une certaine durée $(a)$. Dans ce contexte, on n'insistera jamais assez sur la nécessité, pour les maîtres d'ouvrage et les autres responsables des aménagements, de faire connaître le plus tôt possible les projets susceptibles de se réaliser à plus ou moins court terme, de façon à ce que les stations de mesure hydrologiques et climatologiques adéquates soient mises en place le plus vite possible, afin de fournir des séries de mesures exploitables statistiquement au moment de l'élaboration des projets d'exécution.

\section{Remarques diverses sur les modèles probabilistes}

On fait souvent le reproche, plus ou moins explicite, aux modèles probabilistes, de constituer des schémas simplistes, témoignant de l'ignorance de leur auteur en ce qui concerne les phénomènes physiques en jeu. Ceci conduit à faire les observations suivantes:

- peu importe qu'un modèle soit simpliste, du moment qu'il comporte les éléments nécessaires à la résolution des problèmes pour lesquels il est établi;

-- dans cet ordre d'idées, rien n'oblige à ce qu'un seul et même modèle réponde à deux types de préoccupations, d'une part celles de «l'ingénieur» qui, à court terme, n'est généralement intéressé que par certains termes du bilan hydrologique exprimés en un petit nombre de stations d'observations, en fonction du problème qu'il doit résoudre; d'autre part, celles du «savant» qui cherche une explication aussi cohérente et complète que

(a) Et, bien entendu, d'une qualité aussi bonne que possible, mais cette dernière exigence relative aux données n’est pas spécifique de leur exploitation statistique. possible des phénomènes physiques observés; en d'autres termes, on peut très bien dissocier l'exploitation des résultats et la recherche des explications;

- l'auteur de modèles probabilistes n'est pas forcément dupe de ses propres schémas, au point de croire qu'ils sont la réalité même: dans le domaine hydrologique, une «loi » de probabilité ne constitue même pas une hypothèse sur la probabilité réelle du phénomène, mais une simple image destinée à formaliser mathématiquement une distribution de fréquence observée; partant de là un modèle probabiliste ne prétend constituer qu'un outil de calcul, ni plus ni moins.

\section{Interpénétration avec les modèles physiques}

Le fait que, généralement, les phénomènes physiques n'interviennent pas explicitement dans les modèles probabilistes ne signifie pas nécessairement que l'auteur de tels modèles ignore la réalité physique sous-jacente aux données qu'il manipule; au contraire, cette réalité physique est un guide essentiel dans le choix des relations probabilistes, guide d'autant plus précieux que les données sont en petit nombre, et qu'avec les seuls critères statistiques, on risquerait de retenir des hypothèses physiquement aberrantes, le risque étant d'autant plus important que les données portent sur de plus courtes séries.

La frontière entre modèles probabilistes et déterministes est d'ailleurs de plus en plus floue et les modèles mixtes sont fréquents. Il est bien certain qu'au fur et à mesure de l'amélioration de l'information, la part du "probabiliste » tendra à se résorber au profit du «déterministe». A la limite, il est d'ailleurs possible de concevoir ce que seraient des modèles d'usage universel utilisant une information très riche: il s'agirait de modèles physiques de bassins, dans lesquels les différents termes du bilan hydrologique seraient complétés par des résidus aléatoires représentant les erreurs accidentelles de mesure et la différence existant entre les grandeurs physiques et leur estimation (en particulier la différence entre la lame d'eau pluviale sur un bassin et les hauteurs pluviométriques ponctuelles utilisées pour l'estimer).

En attendant que la mise au point de tels modèles soit suffisamment avancée pour les rendre opérationnels (dans la mesure où l'information hydrologique et climatologique permettra de les mettre effectivement en œuvre), il y a lieu de se contenter d'utiliser une gamme de différents types de modèles, dont chacun est plus spécialement adapté à un genre de problème particulier et à l'information disponible pour le résoudre.

\section{Remerciements}

Nous tenons à exprimer notre gratitude à $M$. Hlavek, Ingénieur en Chef du Génie Rural, des Eaux et des Forêts, dont les suggestions ont permis d'améliorer très sensiblement la rédaction du présent article. 


\section{Bibliographie}

[1] Bernier (J.). - Problèmes et méthodes de l'hydrologie statistique. Bulletin du Centre de Recherches et d'Essais de Chatou, $\mathrm{n}^{\circ} 4$ (juin 1963).

[2] Dauty (J.). - «Les débits mensuels. Simulation et exploitation pour la gestion des ressources ». Thèse, Université des Sciences et Techniques du Languedoc, Montpellier (1972).

[3] Filering (M. B.). - Multivariate technique for synthetic hydrology. Journal of the Hydraulics Division, Procedings A.S.C.E., 90, HY 5 (1964).

[4] Hammersley (J. M.) et Handscomb (D. S.). - «Monte-Carlo methods». John Wiley and sons, New York (1964).

[5] Lugiez (F.) et Guirlor (P.). - La prévision des débits et des crues dans les services d'exploitation d'Electricité de France. "X Journées de 1'Hydraulique », Question II, Rapport 7, Société Hydrotechnique de France, Paris (1968).

[6] Mougin (G.) et Dujardin (J. M.). - «Les modèles déterministes de transformation précipitation-débit ». (La Houille Blanche, $\mathrm{n}^{\circ} 4$ (1972).

[7] Tiercelin (J. R.). - «Paramètres régionaux relatifs aux ressources en eau. Utilisation. Précision d'estimation ». Colloque sur l'élaboration des projets d'utilisation des ressources en eau sans données suffisantes. Madrid (1973). 